\title{
Towards a global initiative for fibrosis treatment (G\|FT)
}

\author{
Maria Molina-Molina ${ }^{1,2}$, Alvar Agusti $^{2,3}$, Bruno Crestani ${ }^{4}$, David A. Schwartz ${ }^{5}$, \\ Melanie Königshoff (10 ${ }^{6}$, Rachel C. Chambers ${ }^{7}$, Toby M. Maher ${ }^{8,9}$, Rosa Faner ${ }^{2,3}$, \\ Ana Lucia Mora ${ }^{10,11}$, Mauricio Rojas ${ }^{10,12}$, Katerina M. Antoniou ${ }^{13}$ and \\ Jacobo Sellares 2,3
}

Affiliations: 'Servei de Pneumologia, Laboratori de Pneumologia Experimental, IDIBELL, Campus de Bellvitge, Universitat de Barcelona, Barcelona, Spain. ${ }^{2}$ CIBER of Respiratory Diseases, ISCIII, Barcelona, Spain. ${ }^{3}$ Servei de Pneumologia, Institut Respiratori, Hospital Clinic, Universitat de Barcelona, IDIBAPS, Barcelona, Spain. ${ }^{4}$ Service de Pneumologie A, Hospital Bichat, University Paris Diderot, Paris, France. ${ }^{5}$ Dept of Medicine, University of Colorado, Aurora, CO, USA. ${ }^{6}$ Division of Pulmonary Sciences and Critical Care Medicine, Dept of Medicine, University of Colorado, Aurora, CO, USA. ${ }^{7}$ Centre for Inflammation and Tissue Repair, UCL Respiratory, University College London, London, UK. ${ }^{8}$ Interstitial Lung Disease Unit, Royal Brompton and Harefield NHS Foundation Trust, London, UK. ${ }^{\prime}$ Fibrosis Research Group, National Heart and Lung Institute, Imperial College, London, UK. ${ }^{10}$ Division of Pulmonary, Allergy, and Critical Care Medicine, University of Pittsburgh, Pittsburgh, PA, USA. ${ }^{11}$ Vascular Medicine Institute, University of Pittsburgh, Pittsburgh, PA, USA. ${ }^{12}$ The Dorothy P. and Richard P. Simmons Center for Interstitial Lung Disease, University of Pittsburgh, Pittsburgh, PA, USA. ${ }^{13}$ Dept of Respiratory Medicine and Laboratory of Molecular and Cellular Pneumonology, Medical School, University of Crete, Heraklion, Greece.

Correspondence: Maria Molina Molina, Servei de Pneumologia, Laboratori de Pneumologia Experimental, IDIBELL, Campus de Bellvitge, Universitat de Barcelona, Barcelona, Spain.

E-mail: mariamolinamolinaahotmail.com

ABSTRACT Idiopathic pulmonary fibrosis (IPF) is a progressive lung disease characterised by increased scarring of lung tissue. Despite the recent introduction of novel drugs that slow disease progression, IPF remains a deadly disease, and the benefits of these new drugs differ markedly between patients.

Human diseases arise due to alterations in an almost limitless network of interconnected genes, proteins, metabolites, cells and tissues, in direct relationship with a continuously changing macro- or microenvironment. Systems biology is a novel research strategy that seeks to understand the structure and behaviour of the so-called "emergent properties" of complex systems, such as those involved in disease pathogenesis, which are most often overlooked when just one element of disease pathogenesis is observed in isolation.

This article summarises the debate that took place during a European Respiratory Society research seminar in Barcelona, Spain on December 15-16, 2016, which focused on how systems biology could generate new data by integrating the different IPF pathogenic levels of complexity. The main conclusion of the seminar was to create a global initiative to improve IPF outcomes by integrating cutting-edge international research that leverages systems biology to develop a precision medicine approach to tackle this devastating disease.

@ERSpublications

A novel call to action for implementing systems biology in IPF research http://ow.ly/Is0A30gpnVb

Cite this article as: Molina-Molina M, Agusti A, Crestani B, et al. Towards a global initiative for fibrosis treatment (GIFT). ERJ Open Res 2017; 3: 00106-2017 [https://doi.org/10.1183/23120541.00106-2017].

Received: Aug 252017 | Accepted: Oct 062017

Copyright $\odot$ ERS 2017. This article is open access and distributed under the terms of the Creative Commons Attribution Non-Commercial Licence 4.0. 


\section{Introduction}

Idiopathic pulmonary fibrosis (IPF) is a progressive disease characterised by increased scarring of lung tissue [1]. Despite the recent introduction of novel drugs that slow disease progression (namely pirfenidone and nintedanib), IPF remains a deadly disease, and the benefits of these new drugs differ markedly between patients [2]. Furthermore, additional anti-fibrotic compounds have failed to demonstrate efficacy in IPF, probably because of limitations in the translation of pre-clinical studies into humans (figure 1) [3]. To improve the prognosis of individual IPF patients it is essential to move the field toward a personalised medicine approach [4]. In this context, we need to better understand the multilevel, network-based biological complexity of IPF (figure 2). Systems biology is a novel research strategy that seeks, precisely, to understand the so-called "emergent properties" of complex biological systems (such as clinical presentation or treatment response) and to understand disease heterogeneity by identifying links and assessing individual risk of developing a disease at different network levels (molecular, cellular, clinical and environmental) [5]. Thus, systems biology is ideally suited to move the field of IPF towards a most needed precision medicine scenario.

To address these issues, the Scientific Committee of the European Respiratory Society (ERS), in collaboration with the ERS group on diffuse parenchymal lung disease, organised a Research Seminar in Barcelona, Spain on December 15-16, 2016 (http://www.ers-education.org/events/research-seminars/ integrating-systems-biology-approach-in-idiopathic-pulmonary-fibrosis-research,-barcelona-2016.aspx).

The major conclusion of this seminar was the need to design, organise, launch and support a global initiative for fibrosis treatment (GIFT), based on international translational research collaboration and a comprehensive, systems-biology based approach to the disease. An organisation like GIFT would have the potential to move the field of IPF forward quickly and effectively to improve understanding and to identify an effective treatment. This report summarises the topics discussed durign this research seminar that lead to the proposal for GIFT.

\section{Current research in IPF: the need to integrate multi-level network complexity}

Figure 2 presents a graphical representation of four networks (genetic, cellular, metabolic and environmental) with potential impacts not only on disease development, progression and response to therapy, but also to the different variants or phenotypes of the disease. Dissecting and integrating them is essential to establish a better understanding of the disease and for development of novel therapies.
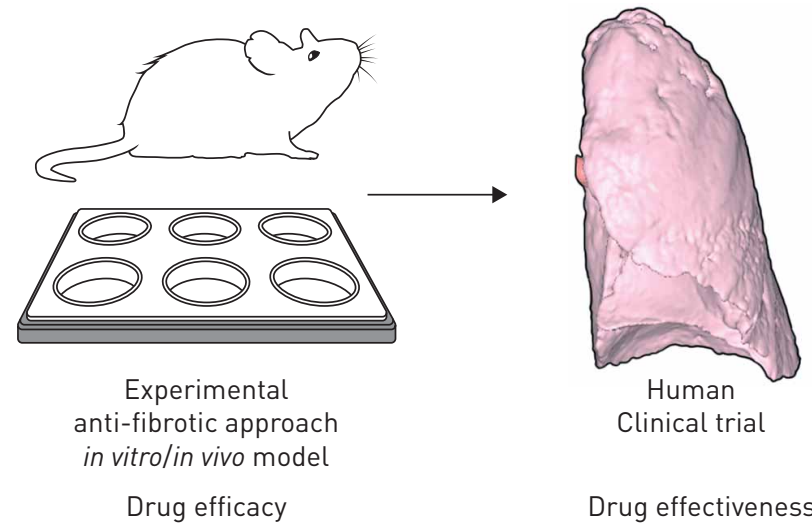

Drug efficacy

Drug effectiveness

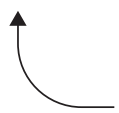

$$
\begin{aligned}
& \text { Why? } \\
& \text { Systems biology } \\
& \text { of IPF complexity }
\end{aligned}
$$

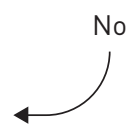

FIGURE 1 Systems biology as a tool to improve idiopathic pulmonary fibrosis (IPF) treatment effectiveness. Harnessing the power of systems biology may provide explanations for discrepancies between in vitro/in vivo experiments and clinical trial results. These indicators can then be incorporated into more finely tuned experiments, which should result in more effective treatments.

Support statement: Supported by European Respiratory Society, Hoffman-La Roche, Boehringher Ingelheim, Linde, Barcelona Respiratory Network (BRN). Toby Maher is supported by an NIHR Clinician Scientist Fellowship (NIHR Ref CS-2013-13-017). Rosa Faner is supported by a Miguel Sevet I Fellowship, CP16/00039. Funding information for this article has been deposited with the Crossref Funder Registry

Conflict of interest: Disclosures can be found alongside this article at openres.ersjournals.com 


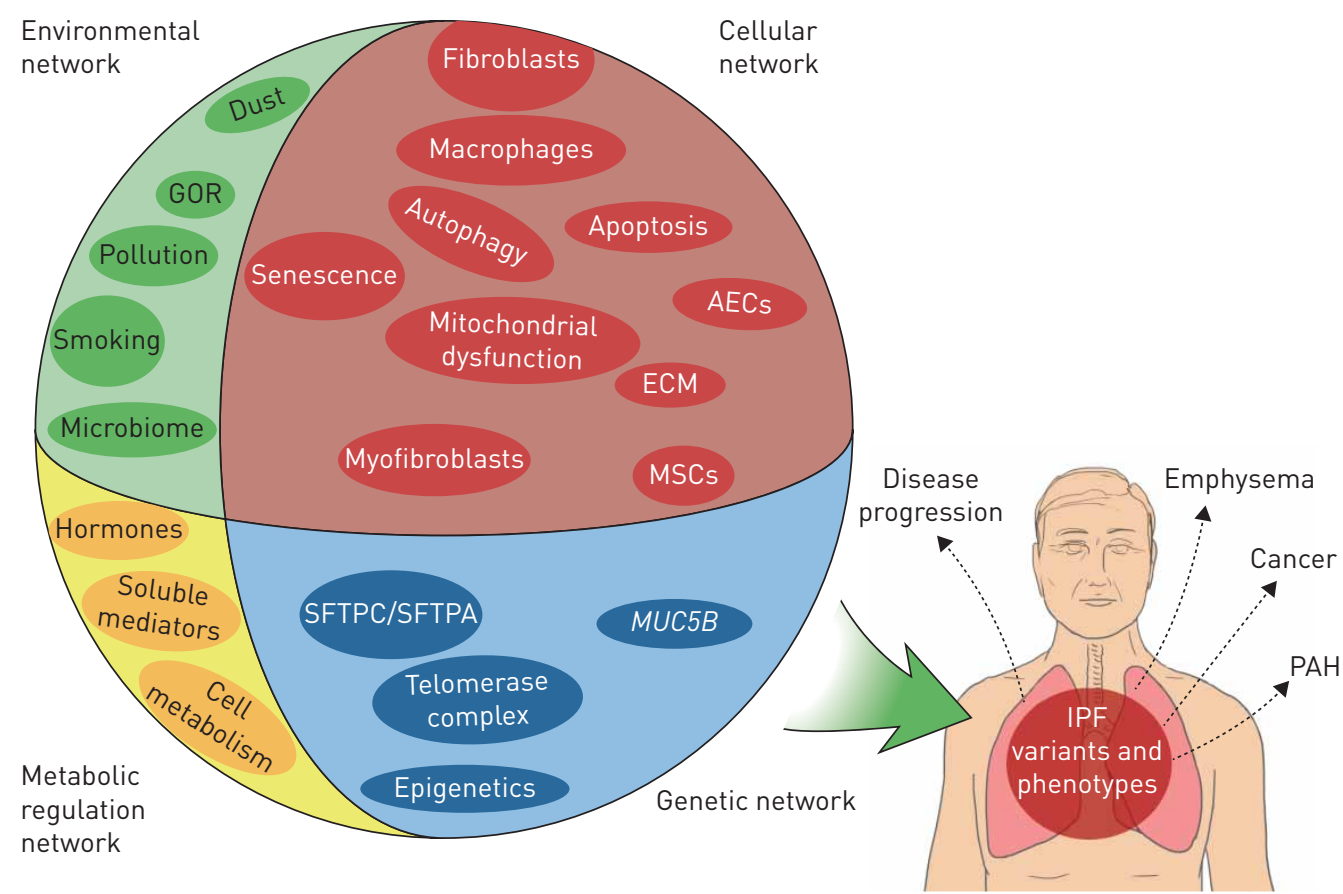

FIGURE 2 Translation from endotype to phenotype, variants and comorbidities. Four main biological networks are involved in idiopathic pulmonary fibrosis (IPF) that include several key elements of the accelerated ageing and lead to the consequent altered wound healing. 1) Cellular network: mesenchymal stem cells (MSCs), alveolar epithelial cells (AECs), extracellular matrix (ECM), fibroblasts, myofibroblasts, macrophages, senescence, mitochondrial dysfunction, apoptosis, autophagy. 2) Metabolic regulation network: hormones, soluble mediators, cell metabolism. 3) Genetic network: MUC5B, SFTPC/SFTPA, telomerase complex, epigenetics. 4) Environmental network: smoking, pollution, gastro-oesophageal reflux (GOR), dust, microbiome. The interconnection among the different players and the clinical repercussions of this complex integrative model is not completely understood. Systems biology is probably the perfect tool to finally shed light on these elusive questions. PAH: pulmonary arterial hypertension.

\section{Genetic network}

IPF is a complex genetic disorder [6-8]. At least eight different genes (MUC5B, TERT, TERC, RTEL1, PARN, SFTPC, SFTPA1 and SFTPA2) and 11 gene variants in novel loci have been associated with the disease [9]. A common gain-of-function promoter variant in the MUC5B gene (rs35705950) accounts for $30-35 \%$ of the risk for developing IPF and can potentially help identify subjects with a higher risk or patients with preclinical pulmonary fibrosis [9]. Mechanistically, this MUC5B variant appears to decrease mucociliary clearance, which can then enhance cell injury and alter wound repair [9].

Another important hub in the genetic IPF network relates to mechanisms of cell ageing. The discovery of telomerase and telomere functions received the Nobel Prize in Medicine in 2009 [10], and the role of telomerase and telomere attrition in ageing and other diseases has been well established [11, 12]. Telomere length decreases with cell division (i.e. with physiological ageing). If telomeres are shortened, cells age. Conversely, if telomerase activity is high, telomere length is better maintained and cellular senescence is delayed. For instance, this is the case for cancer cells, which can be considered immortal. In IPF, telomerase gene mutations and reduced telomere length are highly prevalent [13, 14]. Telomerase dysfunction and telomere shortening are associated with increased cell senescence and cell damage [15]. Interestingly, telomerase gene mutations have been associated with poor prognosis in IPF [13, 14], and the severity of telomere attrition correlates with disease progression and outcome [16]. Further, age-related cell perturbations found in epithelial cells and fibroblasts of patients with IPF are not present in normal lungs of similar age individuals [17]. However, while it is well established that familial forms of IPF are linked to dysfunctional telomerase activity and mutations in surfactant proteins, the relationship of ageing to the majority of sporadic IPF cases is currently under study. Finally, in addition to telomere attrition, there are other genetic footprints associated with accelerated ageing, including genomic instability and epigenetic changes [18], which can also be relevant to the pathobiology of IPF. Interventions to modify these age-related characteristics are currently being investigated in IPF [19], since the lung-ageing process appears to be associated with the generation of a vulnerable alveolar epithelium, as well as a reduction of enzymes involved in telomere maintenance [20]. 
Cellular network

Traditionally, the two cellular actors considered most relevant in IPF were alveolar epithelial cells (AECs) and myofibroblasts, respectively. Recent research applying single cell sequencing approaches provides evidence for the existence of several subpopulations of AECs and a variety of interstitial fibroblasts and other mesenchymal cells. Furthermore, mesenchymal stem cells (MSCs) have also been shown to be important cellular actors involved in the pathogenesis of lung fibrosis.

AECs are essential for normal lung function, forming the tight functional barrier required for gas exchange [21]. Under normal conditions, alveolar type II (ATII) cells act as progenitor cells that initiate alveolar epithelial repair and restoration, giving rise to either new ATII cells or differentiation into alveolar type I cells [22]. However, activated AEC's can participate in the fibrogenic response by secreting mesenchymal proteins [23]. In IPF, reprogramming of these cells results in a number of different phenotypes and functions, which include proliferation and bronchiolisation, apoptosis, and acquisition of mesenchymal features $[24,25]$.

Fibroblasts and myofibroblasts are considered the key effector cells in the fibrogenic response in IPF and a hub within the cellular network because they are responsible for the large amount of ECM production in the fibrotic tissue [26]. Yet, there are still many unanswered questions in relation to fibroblasts. For instance, we do not know if there is only one specific phenotype of fibrotic fibroblast or a variety [27], what the origin may be of the accumulating myofibroblasts in the IPF lung, and the identity of the main pathways driving proliferation and differentiation of these heterogeneous cells [28]. From a network perspective, given that fibroblasts in the alveolus live in close contact with AECs, it is crucial to understand what mechanisms link epithelial injury and fibroproliferation.

With particular importance for the systems biology and cross-network perspective, alveolar macrophages seem to be particularly affected by the pro-fibrotic environment of IPF lungs, which deregulates functionality in wound-healing and repair [29], leading to studies on the downstream implications of high levels of oxidised mitochondria.

Finally, another key consideration relates to the role of MSCs in IPF [30]. As discussed earlier, mechanisms associated with ageing participate in the pathobiology of IPF. Part of this ageing process includes the exhaustion of MSCs and the changes in the function of bone marrow (BM)-MSCs that depend on age $[17,31]$. Interestingly, harvested aged MSCs are less effective in preventing fibrotic changes than MSCs from young animals [32]. In addition, MSCs from IPF patients have smaller mitochondria and undergo accelerated senescence $[18,31]$. The functional and clinical implications of these observations merit further investigation [18].

\section{Metabolic regulation network}

Metabolism is a key player in biological complexity [33]. For example, in cancer it is well established that rapid cell proliferation is associated with increased glucose uptake (which can be detected by increased uptake of 18F-FDG (2-fluoro-2deoxy-D-glucose) on positron emission tomography (PET)) [34]. IPF lungs also present increased uptake of $18 \mathrm{~F}-\mathrm{FDG}[35,36]$, and recent studies have shown that metabolic reprogramming plays a key role in fibroproliferation and myofibroblast differentiation [37, 38]. Classically, several growth factors, cytokines and hormones have been reported to act in cell-to-cell and cell-to-extracellular matrix (ECM) cross-interactions, inducing different cell metabolic and fibrotic changes. Furthermore, mechanical ECM properties, such as increased stiffness, also contribute to influencing cell phenotype. More recently, a number of hallmarks of ageing such as accumulation of misfolded proteins and dysfunctional mitochondria, dysregulation of miRNA expression, and deficient autophagy have been described to contribute to increasing the pro-fibrotic response and modifying cell behaviour and metabolism $[12,18]$.

\section{Environmental network}

Cells and tissues (hence gene, proteins and metabolites) are constantly exposed to dynamic micro- and macro-environmental changes (e.g. smoking, the microbiome, pollution and/or gastro-oesophageal reflux (GOR)) that can modulate their interaction, changing the endotype and, consequently, the phenotype [39-41]. Smoking exposure increases the probability of the associated emphysema. The treatment of GOR is recommended in the updated IPF guidelines since, when present, it may increase AEC cell injury [2, 39]. The most recent advances in the knowledge of microbiome postulate that the bacterial signature induces a host response, which may play a role in disease behaviour and therefore represent another target to prevent or treat [41]. 
FIGURE 3 Iterative working phases of systems biomedicine. Systems biology is a process that becomes highly honed as new information is aggregated, filtered, tested, and allocated as essential data for further cycling through the four steps. Several lines of investigation may result. Reproduced from [44] with permission. (C) Permanyer 2017.

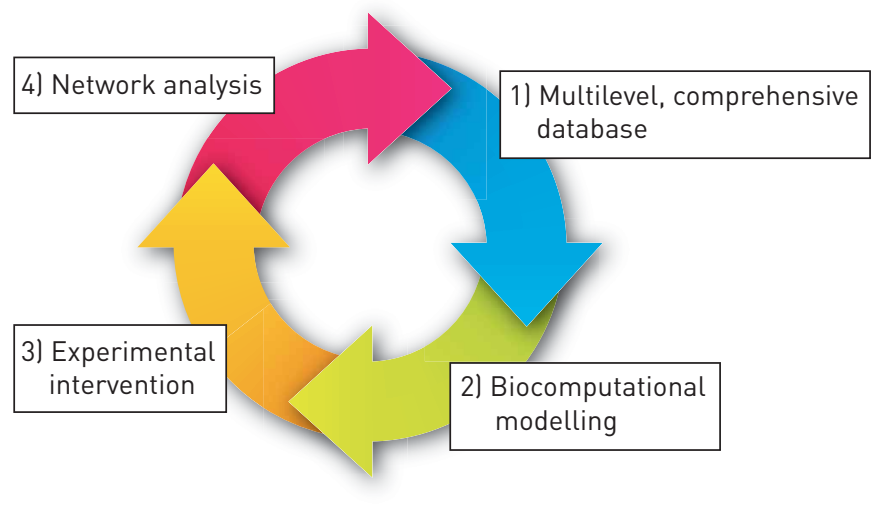

\section{Systems biology: well suited for the complexity of IPF research}

Systems biology is an iterative research strategy (figure 2). The process generally starts with collection of multi-dimensional data (genomics, transcriptomics, metabolomics, microbiomics, etc.). The input variables are rather infinite, but using computational biology, models that aggregate emergent properties can be created and then challenged by experimental intervention. In this context, network analysis is a powerful technique to better visualise complex biological systems using graphs. This process is iterated until the resulting model reflects with sufficient accuracy the experimental findings [42]. SELMAN and PARDO [43] have already used this approach to propose an integral model of IPF in which a combination of gene variants results in a loss of epithelial integrity that, in turn, limits the capacity of alveoli to respond to injury. Thereafter, epigenetic reprogramming takes place and affects epithelial cells and fibroblasts, resulting in destruction of the lung tissue [43].

Following discussions on current research in IPF, participants in the ERS seminar agreed that a systems biology approach has great potential to understand better IPF pathobiology, differences in clinical phenotypes and response to therapy as well as correlations with the clinical expression of IPF variants (figure 3). Yet, in order to achieve these goals the following critical requirements should be considered and implemented.

\section{Standard operating procedures}

Interoperability of diverse data is critical. Current ongoing studies, such as PROFILE [45] and the European IPF network registry [46] collect different biological samples to work collaboratively on IPF diagnosis and monitoring, and are already using a shared set of predefined rigorous standard operating procedures (SOPs).

\section{Cooperative biobank}

Accessing a large number of biological samples sampled and stored according to SOPs, particularly those obtained in the early phases of the disease, has the potential to identify novel key pathobiological mechanisms and biomarkers that facilitate the discovery of new treatments to prevent disease progression. By combining efforts in sample collection at different academic centres, a larger and more comprehensive biobank could be made available to all IPF researchers, after appropriate assessment of the scientific value and priority of the project proposed [47]. The Global IPF Collaborative Network (www.ucdenver.edu/ academics/colleges/medicalschool/departments/medicine/globalipf/Pages/GlobalIPF.aspx) with a DNA consortium is a successful example of this type of initiative [48], with various types of IPF samples (e.g. surgical lung biopsies, cryobiopsies and cryopreserved cells).

\section{Education and support on novel bioinformatics techniques}

Systems biology approaches rely heavily on novel bioinformatics analyses. Most basic and clinical research centres do not yet have the necessary educational framework and bioinformatics expertise in place to take advantage of rapidly evolving bioinformatics approaches, which is also crucial for interpretation of results.

\section{The global initiative for fibrosis treatment (GIFT)}

Participants in the seminar unanimously agreed that an organisation specifically tailored to foster top-notch, cooperative, international IPF research was needed to promote the discovery, development and implementation of more effective and precise (i.e. personalised) treatment options for IPF. To achieve this, it was proposed to connect existing IPF networks across the globe and build upon databases from previous 
initiatives such as the ERS pan-European IPF registry and biobank (ARIANE-IPF) and the eurIPFreg and eurIPFbank (www.pulmonary-fibrosis.net) [46]. In addition, national patient groups could be aligned to move patient treatment forward with equal access and standards as proposed in the European IPF Patient Charter (www.ipfcharter.org). Pharmaceutical and biotechnology partners could also be welcomed. The participants suggested this new body could undertake the directive of setting the standards and regulation needed for an integrated research network across the IPF community that leverages the potential of systems biology [49]. This body could potentially be named the "Global initiative for fibrosis treatment" (GIFT), which could also apply for and allocate specific research funds, establish connections between biobanks to support cutting-edge research, and develop global strategies for IPF challenges.

Participants in the seminar concluded that the next ERS International Congress in Milan (Italy) in September 2017 would be an ideal forum to develop these ideas further, establish specific goals, and determine task allocation (organisation, chairmanship, frequency of meetings) and short-term milestones. The first priority for the proposed body is an inclusive invitation to contribute to this worldwide initiative and to design a governance document, to establish directives, functions, and responsibilities, which was presented at the ERS International Congress in Milan. Secondly, it must be determined by quorum, including all the relevant international experts, how these goals will be achieved. Due to the costly nature of biomedical research, finding funds for the logistic implementation of GIFT is also required. The GIFT initiative was approved by the ILD assembly of the ERS during the ERS International Congress in Milan.

All in all, GIFT would then have the potential to direct and facilitate the highest-quality integrative research with the final goal of finding novel, effective, and safe therapies for this complex and lethal disease.

\section{Acknowledgements}

The authors thank all participants in the Barcelona ERS Research seminar (listed below) for their contribution to the discussion. We are also indebted to the ERS technical organisers for the event coordination.

Participants in the ERS research seminar (December 15-16, 2016, Barcelona, Spain): Aburto Barrenechea M, Asije G, Bibaki E, Bingle C, Bueno M, Bullich Ramon M, Campo Ezquibela A, Campo I, Carleo A, Cruz T, Daccord C, Duitman JW, Eagan T, Eickelberg O, Froidure A, Garcia-Moyano M, Garcia-Nuñez M, Gazdhar A, Homolka J, Hoyer N, Kool M, Korfei M, Koutsopoulos A, Kwakkel-van Erp H, Lofdahl A, Lopez-Rodriguez E, Margaritopoulos G, Lafuente JM, Montes-Worboys A, Morais A, Moreno A, Perona R, Petrek M, Pitozzi V, Planas Cerezales L, Purokivi M, Rivera P, Ruwisch J, Sala E, Schliep R, Shaker SB, Simillion C, Sterclova M, Tomos I, Trachalaki A, Trevisani M, Tsitoura E, Vasaková M, Vasarmidi E, Vicens-Zygmunt V, Wijsenbeek M, Woldhuis R, Wuyts W.

\section{References}

1 Gross TJ, Hunninghake GW. Idiopathic Pulmonary Fibrosis. N Engl J Med 2001; 345: 517-525.

2 Raghu G, Rochwerg B, Zhang Y, et al. An Official ATS/ERS/JRS/ALAT Clinical Practice Guideline: treatment of idiopathic pulmonary fibrosis. An update of the 2011 Clinical Practice Guideline. Am J Respir Crit Care Med 2015; 192: e3-e19.

3 Maher TM. Precision medicine in idiopathic pulmonary fibrosis. QJM 2016; 109: 585-587.

4 Brownell R, Kaminski N, Woodruff PG, et al. Precision medicine: the new frontier in idiopathic pulmonary fibrosis. Am J Respir Crit Care Med 2016; 193: 1213-1218.

5 Agusti A, Sobradillo P, Celli B. Addressing the complexity of chronic obstructive pulmonary disease: from phenotypes and biomarkers to scale-free networks, systems biology, and P4 medicine. Am J Respir Crit Care Med 2011; 183: 1129-1137.

6 Borie R, Kannengiesser C, Sicre de Fontbrune F, et al. Management of suspected monogenic lung fibrosis in a specialised centre. Eur Respir Rev. 2017; 26: 160122.

7 Kropski JA, Young LR, Cogan JD, et al. Genetic evaluation and testing of patients and families with idiopathic pulmonary fibrosis. Am J Respir Crit Care Med 2017; 195: 1423-1428.

8 Kannengiesser C, Borie R, Ménard C, et al. Heterozygous RTEL1 mutations are associated with familial pulmonary fibrosis. Eur Respir J 2015; 46: 474-485.

9 Evans CM, Fingerlin TE, Schwarz MI, et al. Idiopathic pulmonary fibrosis: a genetic disease that involves mucociliary dysfunction of the peripheral airways. Physiol Rev 2016; 96: 1567-1591.

10 Nobelprize.org. The Nobel Prize in Physiology or Medicine 2009 - Press Release. www.nobelprize.org/nobel prizes/medicine/laureates/2009/press.html Date last updated: October 5, 2009. Date last accessed: March 2, 2017.

11 López-Otín C, Blasco MA, Partridge L, et al. The hallmarks of aging. Cell 2013; 153: 1194-1217.

12 Meiners S, Eickelberg O, Königshoff M. Hallmarks of the ageing lung. Eur Respir J 2015; 45: 807-827.

13 Stuart BD, Lee JS, Kozlitina J, et al. Effect of telomere length on survival in patients with idiopathic pulmonary fibrosis: an observational cohort study with independent validation. Lancet Respir Med 2014; 2: 557-565.

14 Molina-Molina M, Xaubet A, Li X, et al. Angiotensinogen gene G-6A polymorphism influences idiopathic pulmonary fibrosis disease progression. Eur Respir J 2008; 32: 1004-1008.

15 Alder JK, Barkauskas CE, Limjunyawong N, et al. Telomere dysfunction causes alveolar stem cell failure. Proc Natl Acad Sci USA 2015; 112: 5099-5104.

16 Planas L, Arias-Salgado EG, Buendia-Roldán I, et al. Clinical predictive factors and prognostic implications of telomere shortening in sporadic and familial idiopathic pulmonary fibrosis. Poster session presented at: ERS Research Seminar: Integrating Systems Biology Approach in Idiopathic Pulmonary Fibrosis Research. December 15-16, 2016.

17 Kapetanaki MG, Mora AL, Rojas M. Influence of age on wound healing and fibrosis. J Pathol 2013; 229: 310-322.

18 Mora AL, Bueno M, Rojas M. Mitochondria in the spotlight of aging and idiopathic pulmonary fibrosis. J Clin Invest 2017; 127: 405-414 
19 Borok Z. Alveolar epithelium: beyond the barrier. Am J Respir Cell Mol Biol 2014; 50: 853-856.

20 Korfei M, Klaus D, Stelmaszek D, et al. Lung ageing in C57/Bl6 and BALB/c mice is characterized by a defective surfactant metabolism, increased ER stress and inflammasome induction. Poster session presented at: ERS Research Seminar: Integrating Systems Biology Approach in Idiopathic Pulmonary Fibrosis Research. December 15-16, 2016.

21 Camelo A, Dunmore R, Sleeman MA, et al. The epithelium in idiopathic pulmonary fibrosis: breaking the barrier. Front Pharmacol 2013; 4: 173.

22 Barkauskas CE, Cronce MJ, Rackley CR, et al. Type 2 alveolar cells are stem cells in adult lung. J Clin Invest 2013; 123: 3025-3036.

23 Yang J, Wheeler SE, Velikoff M, et al. Activated alveolar epithelial cells initiate fibrosis through secretion of mesenchymal proteins. Am J Pathol 2013; 183: 1559-1570.

24 Königshoff M. Lung cancer in pulmonary fibrosis: tales of epithelial cell plasticity. Respiration 2011; 81: 353-358.

25 Königshoff M, Eickelberg O. WNT signaling in lung disease: a failure or a regeneration signal? Am J Respir Cell Mol Biol 2010; 42: 21-31.

26 Bochaton-Piallat ML, Gabbiani G, Hinz B. The myofibroblast in wound healing and fibrosis: answered and unanswered questions. F1000Research 2016; 5: 752.

27 Barkauskas CE, Noble PW. Cellular mechanisms of tissue fibrosis. 7. New insights into the cellular mechanisms of pulmonary fibrosis. Am J Physiol Cell Physiol 2014; 306: C987-C996.

28 O'Reilly S. Epigenetics in fibrosis. Mol Aspects Med 2016; 54: 89-102.

29 Athina T, Eirini V, Eliza T, et al. Alveolar macrophages show elevated oxidized mitochondria levels in IPF Autophagy, mitophagy and telomere length implications. Poster session presented at: ERS Research Seminar: Integrating Systems Biology Approach in Idiopathic Pulmonary Fibrosis Research. December 15-16, 2016.

30 Cruz T, López-Giraldo A, Agustí A, et al. Quantification and characterization of human lung mesenchymal stem cells. Poster session presented at: ERS Research Seminar: Integrating Systems Biology Approach in Idiopathic Pulmonary Fibrosis Research. December 15-16, 2016.

31 Faner R, Rojas M, Macnee W, et al. Abnormal lung aging in chronic obstructive pulmonary disease and idiopathic pulmonary fibrosis. Am J Respir Crit Care Med 2012; 186: 306-313.

32 Wecht S, Rojas M. Mesenchymal stem cells in the treatment of chronic lung disease. Respirology 2016; 21: 1366-1375.

33 Barabási AL, Gulbahce N, Loscalzo J. Network medicine: a network-based approach to human disease. Nat Rev Genet 2011; 12: 56-68.

34 Vander Heiden MG, Cantley LC, Thompson CB. Understanding the Warburg effect: the metabolic requirements of cell proliferation. Science 2009; 324: 1029-1033.

35 Win T, Thomas BA, Lambrou T, et al. Areas of normal pulmonary parenchyma on HRCT exhibit increased FDG PET signal in IPF patients. Eur J Nucl Med Mol Imaging 2014; 41: 337-342.

36 Justet A, Laurent-Bellue A, Thabut G, et al. [18F]FDG PET/CT predicts progression-free survival in patients with idiopathic pulmonary fibrosis. Respir Res 2017; 18: 74.

37 Vancheri C, Failla M, Crimi N, et al. Idiopathic pulmonary fibrosis: a disease with similarities and links to cancer biology. Eur Respir J 2010; 35: 496-504.

38 Xie N, Tan Z, Banerjee S, et al. Glycolytic reprogramming in myofibroblast differentiation and lung fibrosis. Am J Respir Crit Care Med 2015; 192: 1462-1474.

39 Raghu G. The role of gastroesophageal reflux in idiopathic pulmonary fibrosis. Am J Med 2003; 115: Suppl. 3A, 60S-64S.

40 Koga Y, Satoh T, Kaira K, et al. Inhaled inorganic elemental analysis of idiopathic pulmonary fibrosis by in-air microparticle induced x-ray emission analysis. Am J Respir Crit Care Med 2016; 193: A2052.

41 Molyneaux PL, Willis Owen SA, Cox MJ, et al. Host-microbial interactions in idiopathic pulmonary fibrosis. Am J Respir Crit Care Med 2017; 195: 1640-1650.

42 Diez D, Agusti A, Wheelock CE. Network analysis in the investigation of chronic respiratory diseases: from basics to application. Am J Respir Crit Care Med 2014; 190: 981-988.

43 Selman M, Pardo A. Revealing the pathogenic and aging-related mechanisms of the enigmatic idiopathic pulmonary fibrosis. An integral model. Am J Respir Crit Care Med 2014; 189: 1161-1172.

44 Agustí A, López-Giraldo A, Cruz T, et al. Relevance of systems biology to respiratory medicine. BRN Rev 2016; 2: $1-13$.

45 Maher T. PROFILEing idiopathic pulmonary fibrosis: rethinking biomarker discovery. Eur Respir Rev 2013; 22: $148-152$.

46 Guenther A, European IPFNetwork. The European IPF Network: towards better care for a dreadful disease. Eur Respir J 2011; 37: 747-748.

47 Villena C, Pozo F, Barberà JA, et al. The CIBERES Pulmonary Biobank Consortium: an opportunity for cooperative international respiratory research. Eur Respir J 2011; 37: 204-206.

48 Yang IV. Epigenomics of idiopathic pulmonary fibrosis. Epigenomics 2012; 4: 195-203.

49 Agusti A, Anto JM, Auffray C, et al. Personalized respiratory medicine: Exploring the horizon, addressing the issues. Am J Respir Crit Care Med 2015; 191: 391-401. 